\title{
Análisis de las características del sistema de clasificación de la Biblioteca del Congreso de los Estados U nidos
}

\author{
LINA ESCALONA Ríos \\ CentroUniversitario deInvestigacionesBibliotecológicas \\ de la UNAM, 04510, México D.F., Tel:56- 23- 03- 29. \\ E-mail:escalona@cuib.laborales.unam.mx
}

\begin{abstract}
RESUMEN
ElSistemadeClasificación delaBiblio tecadelCongreso delosEstadosUni dos de Norteamérica ha sido adoptado por innumerables bibliotecas -especialmentelasuniversitarias-yespecíficamenteen el caso deMéxico, el Siste maesusado en más de280institu cio nesbiblio tecarias; sin em bargo enlamayoría de las instituciones en las que se usa se desconoce la estructura del mismo, lo cuallimitasuutilidad. Enestetrabajo seanalizanlascaracterísticas generalesdel sistema, con objeto deam pliarelám bito deac ción delospro fe sionales de la bibliotecología aplicarlo a las diversas instituciones bibliotecarias. Se proporciona una visión general so breel sistemayel uso delastablas auxiliares, que son elementos fundamentales de cada clase.

Pa la bras cla ve: Cla sifica ción de la Biblio te ca del Congre so, Sis te mas de cla sifica ción
\end{abstract}

\section{ChARACTERISTICSOF THE CLASSifiCATION SYSTEM OF THE LIBRARY OF CONGRESS. AN ANALYSIS LINA E SCALONA-Ríos}

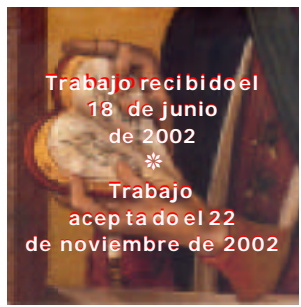

\begin{abstract}
TheUSLibrary of Con gressClassification System has been adop tedbymany libraries, above all in the universities. Specifically, in the case of Mexico the sys tem is in use in more than 280 libraryinstitu tions. Howevermost of the se usersigno rethestruc tu re of theSystem and this con dition hin dersitspro per use. This paperanalyzes thegeneral charac teris tics of the Sys tem in or derto broaden the scope of library science professionals and to apply it in diverse institutions. Itsin ten tionisto giveageneralview of theuse of au xiliarytables, which are essential for each of the classes.

Key Words: Li brary of Con gress Sys tem. Cla sification Sys tems
\end{abstract}

\section{INTRODUCCIÓN}

D esdequeelhom bretuvo lanecesi dad decrearmaterialesdon detu vieseregis tradalainformación, tuvo también queorganizaresosmateriales, sien do ésta una preocupación constante porque de dicha organización depende la recuperación deinformación do cumen tal quesirvecomo baseparalageneración denuevo conocimiento. 
A lo largo delahisto ria, y con baseen lanecesi dad deorganizar, sehan creado di versossistemasdeclasificación, algunosconbasesfilo só ficasyotrosconfinesmeramenteprácticos. Estossistemashansido estu diados, analizadoseim plantadosen di ferentes instituciones bibliotecarias, ya sea adaptándolos o adoptándolos. Tal es el caso del SistemadeClasificación delaBiblio tecadelCon greso deWashington, que ha sido adoptado por innumerables bibliotecas -especialmente las universitarias- y específicamente en el caso de México, el Sistemaesusado en másde280institu cionesbiblio tecarias. Sinembargo, el SistemadeClasificación LC fueideado parare solverunapro blemáticaparticular(laorganización delaco lección delaBiblio tecadel Congreso delosEstadosUnidosdeAmérica)yen unidio madiferen teal español;lo quehacequelos biblio tecariosasuman con ciertasreservassuuso o adap tación para lasnecesidadeslo calesoregionalesdesuspropiasunidadesdeinformación.

Sin em bargo, hayqueconsiderarqueel SistemadeClasificaciónLC obedeceauna estructuraquepuedeseradaptadaymodificadaparaaten derrequerimientosparticulares, paralo cual setieneprimero queen ten derdichaestructu raysuformadeaplicacióngeneral.D ado lo an terior, el objetivo deestetrabajo es analizarlascaracterísiticas principales del Sistema de Clasificación LC, proporcionando una introducción paracomprenderloyusarloadecuadamente.

Parael análisis setomó como baseel trabajo realizado porImmro th's' ${ }^{1}$ ya que es de excelente calidad y cuya consulta es básica para quien desee conocer el Sistema. Porotraparte,ypen san do en el con tex to nacio nal, en el queno to dostienen ac ceso a la ver sión deLC en disco com pac to, secon si derólaversiónim presaquesetienedis po nibleen elCUIB to man do en cuentaquelascaracterísticas del sistemason lasmis masimpresaso enformaelectrónica.

Para lograr lo anterior, el trabajo inicia mencionando brevemente la historia del SistemadeClasificación, po niendo énfasisen eltrabajo realizado porlosbiblio tecariosJamesC.Han son yCharles Martell.D espuésseabordalaestructu rageneral del Sistema de Clasificación LC, su notación y algunos ejemplos para facilitar su comprensión; posteriormen tesemuestran los diversostiposdetablasquecontieneysu formadeaplicarlas. Cabeseñalarque sehaceén fasis en el uso delastablasporquela comprensióndeellasvaapermitirunmejoraprovechamientodelSistema.

Finalmente se dan una serie de conclusiones y recomendaciones que se esperan seandeutilidadparacontinuarcontrabajosdeestanaturaleza.

\section{BREVE H IST O RIA DE L SISTE MA DE CLASIFICACIÓN DE LABIBLIOTECA DEL CONGRESODE EST ADOS UNIDOS (LC)}

Hacia prin cipios delSiglo X IX, parasatisfacerlasnecesidadesdein formaciónbi bliográfica de los miembros del Congreso, el cuerpo legislativo de Estados Unidos 
usaba las colecciones de la Library Company of Philadelphia y de la New Y ork Society Library, pues no contaban con ninguna institución bibliotecaria que apoyara susactividades.Lasco leccio nesdeesasbiblio tecasfueron to madascomo basepara formarlacoleccióndelaBibliotecadelCongreso.

Sin embargo la necesidad de una biblioteca era apremian te, un es pacio en el que los legisladores pudieran consultar las obras que ya se poseían. A esta necesidad se unió el cam bio delasededepo deresen Washingtonyen laquin tasec ción del "An act to makefurtherpro vision fortheremo val and accommo dation of the Go vernment of theUnited States" 2 seestablecelaBiblio tecadelCongreso (LibraryofCongress), y el Presiden te John A dams señalaque se pro vee dela suma de \$5000 dó la res parala compradeloslibrosquefuerannecesariosparaelCongreso. ${ }^{3}$

Conlacreación delaBiblio tecadel Con greso senom braasuprimerbiblio tecario quefueJohn Beckleyyseledesignaun lu garden tro del Capito lio paraqueseordena ranlasco leccio nes. Sinnin gunain formación relacio nadaconlaorganización decolecciones, el personal organizó el acervo por tamaños: en folios, cuartos, octavoso duo décimos,yden tro delosblo quesportamaño, tambiénlo subarregló pornúmero deadquisición,sinconsiderarlatemáticaqueconteníanlosdocumentos.

Elprimercam bio en estaorganización delacolección aparecehacia1808,yacon el tercercatálo go delaBiblio tecaen el queseordenabasegúnel tipo dematerial; esdecir porplanos,leyes, periódicosdelaCasadeRepresentantesdeEstadosUnidos, reportes deCo mitésdelaCasa, reportesejecutivosydo cumentos, recibosyex pedientes, libros decon tabili dad delaCasay gacetas. Estesistemaseusó hasta1808, año en quesehizo no tarlaineficaciadelsistemayseanalizólaconvenien ciadeagru parlosmateriales de acuerdo con lamateria, porlo queseem pezó ausarel sistemautilizado porlaLibrary Company of Philadelphia, que con tabacon 31 clases. En 1812 laBiblio tecadel Congresoagrupólosmaterialesen18ysonlassiguientes:

1. Historia sagrada

2. Historia eclesiástica

3. Historia civil, incluyendo cronología, biografía, antigüedades, etcétera

4. G eografía y topografía, viajes

5. D erecho

6. Ética o el sistema moral en general; teología y mitología

7. Lógica, retórica y crítica

8. Diccionarios, gramática y economía política, etcétera

9. Política general y local; economía política, etcétera

10. Industria y comercio

11. Tácticas militares y navales

12. Agricultura, economía rural, etcétera

13. Historia natural, filosofía natural y experimental, etcétera

2 John Philip Immroth. Immuth's guidetotheLibrarydCongessClassification p. 2.

3 Cfr. Ibid 
14. Medicina, cirugía y química

15. Poesía y drama; novelas

16. Arte y ciencias; literatura miscelánea

17. Gacetas

18. Mapas, cartas y planos 4

El esquema de Filadelfia tomó como base el sistema de clasificación de Francis Baconconalgunasmo dificacio nesquefueron realizadasporD 'A lembert.Baconha bíadivididoelconocimientoendoscategorías:

a)Conocimientohumano. Queincluáalain formación derivadadelossen tidos, lo que se podía observar, tocar, experimentar.

b) Teología. Q ue consideraba la información derivada de la revelación, es decir la información que había sido transmitida por sueños o percepciones divinas.

Lamentablementeen 1814, el Capito lio sufreel ataquedelosingleses, yseprovo ca un granin cen dio quejun to conel edificio dañaaunaenormecan tidad dematerialesal bergados en la Biblioteca. Para reinstalarla Thomas Jefferson -que había colaborado arduamen teenlacreación delaBiblio teca-leofreceal G o bierno deEstadosUnidossu colección. Jefferson tenía su colección organizada y para clasificarsus6,487vo lúmenes, habíausado un sistemapro pio enelqueseagrupaban 44capítulosconsubdivisionesgeo gráficas. Estesistemafueusado porlabiblio tecadurantemuchosañoshaciéndolealgunasadaptacionesconformelofueconsiderandopertinente. ${ }^{5}$

EnlaúltimadécadadelsigloX IX , sehizo eviden tequeelsistemajefferso niano ya no eraadecuado pararesolverlasnecesidadesoriginadasporel crecimiento delacolección, ademásdequeeledificio del Capito lio yaresultabain su ficien te; sepro cedió entonces a construir uno que fuese exclusivamente para albergar la Biblioteca del Congreso,elcualfueinauguradoel ${ }^{\circ}{ }^{\circ}$ denoviembrede1897. ${ }^{6}$

Considerando,por una parte, el nuevo edificio en el queseteníaquereinstalarla biblio tecay, porlaotra, laineficaciadel sistemaqueseestabausan do, sepen só en la necesidad de crear un nuevo sistema de clasificación que permitiera una organización más funcional del acervo. Esta tarea titánica se asignó a dos personajes clave para la creación del Sistema LC: James C. Hanson (Jefe de la D ivisión de Catalogación)yCharles Martell(JefedeClasificación), quienessededicaronal estu dio delos sistemasmásim portantesdelaépo ca:elsistemadeClasificaciónD ecimal deMelvil D ewey, el EsquemadeHalleylaClasificación Ex pan sivadeCutter.Han sonyMar tell realizaron la evaluación de los diferentes sistemas y decidieron que la Clasificación expansivadeCutterpo dríaservircomo baseparael nuevo sistema, con al gunas modificaciones,comoañadirlenúmerosarábigosparaseñalarlasclases.

4 cfr. Ibid p.3.

5 cfr. Ibid p. 4.

6 cfr.MartínezArellano, Felipe.AlgumosproblemasendmangoyapicaaóndaSistemadbClasificadónLC en mateiales latinoameicanos p. 2. 
LainfluenciadelaClasificacióndeCuttersemanifestó prin cipalmenteen dosas pectos:

a) Para el diseño delos autlineso perfilesdel SistemadeClasificaciónLC, quefueron diseñados por Hanson y;

b) Para la realización de la clase Z, estructurada por Martell.

Setrabajó en estaclaseporqueden tro deellasedesarro llaríael sistemaparalaorganización dematerialesdo cumentales, labiblio grafíaylas disciplinasrelacionadas conlibrosydocumentos.

Hanson, por su parte, trabajó en la adaptación de la Clasificación Expansiva de Cuttercon lafinalidad derealizarlos perfilesdelasclasesdel nuevo sistema, ano tando letrasparadesignaralasclasesy ex pan dién do lascon números, conlo quesecreó unaclasificaciónmixtadeletrasynúmeros.

Cabemen cio narquecadaesquemadelsistemasehadesarro llado deformain divi dual ein depen dien te.Como sepuedeob servar, el objetivo quedio origenal Sistema deClasificación delaBibliotecadelCongreso fueúnicamentepráctico, esdecir, seteníaqueresponderaunanecesidadespecíficadeorganizacióndelaco leccióndeuna biblio tecaen particu lar, ladel Con greso. Portan to no tienecomo basenin gún siste mafilosóficonidelconocimiento.

El sistema tam po co fueideado pararesolverlasnecesidadesdeorganización de otro tipo debiblio tecas, sino quesuestructurayorganizaciónco rrespondenalaresolu ción delasnecesidades delas co leccio nes del Con greso, como esel caso delaclase K deD erecho,entreotras. ${ }^{7}$

Sin embargo el SistemadeClasificaciónLC respondeaunaestructuradecarácter general y puede ser usado o adaptado por cual quieruni dad dein formación parare solver problemas de organización. E sporello queseusaen mu chas biblio tecas, no sólo deEstadosUnidossino también deAméricaLatina. ${ }^{8}$ Un caso típi co es el delaliteratura, en el quelosesquemasdelaClasificación LC señalan un número deCutter paralosescrito resquetienen unapro ducciónliterariacontem pladaenEstadosUni dos, pero lasbiblio tecasmexicanascuentan conunagran cantidad deliteraturadeau to resqueno tienen reco no cimien to en el ex tran jero yaquienesselestienequeasig. nar un número de clasificación y un número de Cutter, aplicando la estructura que marcaelSistemadeClasificaciónLC.

\section{EST RUCTURA DEL SISTEMA DE CLASIFICACIÓN LC}

\section{C aracterísticas generales}

Como yasemen cio nó, el SistemadeClasificaciónLC tieneunano taciónmix tade letras y números: la primera letra expresa las clases; las segundas y terceras letras

7 cfr. Jhon Philip Immroth,. Op at. p.14

8 Considerando lo anterior, la Biblioteca del Congreso ha creado una serie de instrumentos para apo yar a otras ins ti tu cio nes como la obraSubet Catalogingmanual:Shdflisting 
muestran las subclases, mientras que los números proporcionan las subdivisiones queestán estructuradasconnúmerosarábigosprogresivosydejanmuchosnúmeros sinuso parafu turas ex pan sio nes, deacuerdo conlasnecesi dadesquevayan surgiendo enlabiblio teca. También sehaceuso denúmerosdecimalesparallevarunamayor precisión enlajerarquización delco no cimien to.Enmu choscasos, acontinuación de losnúmerossedan números deCutter, paraun temadefinido, paraunaregióno para unautorespecíficoysonasignadosdeacuerdo conlaadaptaciónquelaBiblio tecadel Congreso realizóalsistemadiseñadoporCharlesAmiCutter.

Los esquemasson ac tualizadosatravés delasAdditionsanddhanges que aparecen deformaperió dicaydanaco no cerlosnuevosnúmerosdeclasificacióny/ oloscambiosquehansufrido haciaelinteriordelesquema.

Ladivisióndelosesquemasporclasesysubclases, deacuerdo conladivisiónfísica delSistema, espresentadadelasiguientemanera:

Cuadro 1.

Cla ses y subcla ses del Sis te ma de Cla sifica ción LC

\begin{tabular}{|l|l|l|}
\hline A & General works & Trabajos generales \\
\hline B-BJ & Phylo sopy.Psychology & Filosofía. Psicología \\
\hline $\begin{array}{l}\text { BL,BM, BP, } \\
\text { BQ }\end{array}$ & $\begin{array}{l}\text { Religion: Religions, Hin duism,Jut } \\
\text { daism, Islam, Bud hism. }\end{array}$ & $\begin{array}{l}\text { Religión: Religiones,Hinduismo, } \\
\text { Judaísmo, Islam, Budismo. }\end{array}$ \\
\hline BR-BV & Religion: Christianity, Bible. & Religión: Cristiandad,Biblia. \\
\hline BX & Religion: Christiandenominations. & $\begin{array}{l}\text { Religión: D enominaciones cristia } \\
\text { nas. }\end{array}$ \\
\hline C & Auxiliary Scien ces of History. & Ciencias auxiliaresdelahisto ria. \\
\hline D & History: General and Old World. & $\begin{array}{l}\text { Historia: General y Mundo Anti- } \\
\text { guo }\end{array}$ \\
\hline DJK-DK & $\begin{array}{l}\text { Historyof Eastern Euro pe(Gene } \\
\text { rl), Soviet Union, Poland (1987) }\end{array}$ & $\begin{array}{l}\text { Histo riadel O estedeEuro pa } \\
\text { (G eneral), Union Soviética, Polo- } \\
\text { nia (1987) }\end{array}$ \\
\hline DS & His tory of Asia & Histo riadeAsia \\
\hline DT-DX & $\begin{array}{l}\text { Historyof Africa, Astralia, New } \\
\text { Zealand, etc. }\end{array}$ & $\begin{array}{l}\text { Histo riadeAfrica,Australia, Nue } \\
\text { vaZelan da, etc. }\end{array}$ \\
\hline E-F & History:America & Historia: América \\
\hline G & $\begin{array}{l}\text { Geography. Maps. Anthropology. } \\
\text { Recreation. }\end{array}$ & $\begin{array}{l}\text { Geografía. Mapas. Antropología. } \\
\text { Recreación }\end{array}$ \\
\hline H-HJ & Socialsciences:Economics & Ciencias sociales: Economía \\
\hline HM-HX & Social Sciences:So ciology & Ciencias sociales: Sociología \\
\hline J & Political Science & Ciencia política \\
\hline K & Law (General) & Derecho (G eneral) \\
\hline
\end{tabular}


Cuadro 1.(Cont.)

Clases y subclases del Siste made Clasificación LC

\begin{tabular}{|c|c|c|}
\hline KD & $\begin{array}{l}\text { Law of the United Kingdom and } \\
\text { Ireland }\end{array}$ & $\begin{array}{l}\text { Derecho del Rei no Uni do eIrlan- } \\
\text { da }\end{array}$ \\
\hline \begin{tabular}{|l|} 
KDZ,KG- \\
$\mathrm{KH}$
\end{tabular} & $\begin{array}{l}\text { Law of the Americas, LatinAmeri } \\
\text { ca, and the West Indies. }\end{array}$ & $\begin{array}{l}\text { Derecho delasAméricas,América } \\
\text { Latinay el O estedelas In dias. }\end{array}$ \\
\hline $\mathrm{KE}$ & Law of Canada & DerechodeCanadá \\
\hline $\mathrm{KF}$ & Law of the United States. & D erecho deEstadosUnidos. \\
\hline $\mathrm{KJ}-\mathrm{KKZ}$ & Law of Europe & DerechodeEuropa. \\
\hline KJV-KJW & Law of France & Derecho deFrancia. \\
\hline KK-KKC & Law of Germany & D erechodeAlemania. \\
\hline $\mathrm{L}$ & Education & Educación \\
\hline M & Music and books on Music & Músicaylibrosdemúsica. \\
\hline $\mathrm{N}$ & Fine Arts & BellasArtes \\
\hline P-PZ & LanguageandLiteratureTables. & Lenguaje y Tablas de literatura. \\
\hline P-PA & $\begin{array}{l}\text { Philology, linguistics, classical phi- } \\
\text { lology, Classicalliterature. }\end{array}$ & $\begin{array}{l}\text { Filología, Lingüística,Filología } \\
\text { clásica,Literatura clásica. }\end{array}$ \\
\hline PA & $\begin{array}{l}\text { Supplement: Byzan tineand mo- } \\
\text { dern greek Literature. Medieval and } \\
\text { modern LatinLiterature. }\end{array}$ & $\begin{array}{l}\text { Suplemento: Literaturabizantina y } \\
\text { griega moderna.Literatura medie } \\
\text { val y latina moderna }\end{array}$ \\
\hline PB-PH & Mo dernEuro pean Languages & Lenguajeseuropeos modernos. \\
\hline PG & Russian Literature & Literatura rusa \\
\hline PJ-PK & $\begin{array}{l}\text { Oriental Philology and Literature, } \\
\text { Indo-Iranian Philology and Litera- } \\
\text { ture }\end{array}$ & $\begin{array}{l}\text { Filología y Literatura Oriental, Fi- } \\
\text { lología y Literatura Indo-Iraní }\end{array}$ \\
\hline PL-PM & $\begin{array}{l}\text { Lan guages of Eastern A sia, Africa, } \\
\text { O ceania; Hyperbo rean, In dian, and } \\
\text { artificial Languages. }\end{array}$ & $\begin{array}{l}\text { Len guas del O rien te de Asia, Afri- } \\
\text { ca, O ceanía; Hiperboreal, India y } \\
\text { lenguas artificiales. }\end{array}$ \\
\hline P-PM & $\begin{array}{l}\text { Supplement: Index to Languages } \\
\text { and Dialects. }\end{array}$ & $\begin{array}{l}\text { Suplemento: Índice de len guasy } \\
\text { dialectos. }\end{array}$ \\
\hline $\begin{array}{l}\mathrm{PN}, \mathrm{PR}, \mathrm{PS}, \mathrm{P} \\
\mathrm{Z}\end{array}$ & $\begin{array}{l}\text { G eneral Literature, English and } \\
\text { American Literature,Fiction in En- } \\
\text { glish, JuvenileBelles Lettres. }\end{array}$ & $\begin{array}{l}\text { Literatura G eneral,Literatura in- } \\
\text { glesa y Americana,Novela en In- } \\
\text { glés, Novela juvenil. }\end{array}$ \\
\hline $\mathrm{PQ}$ & Part. 1: French literature. & Parte 1. Literatura Francesa. \\
\hline PQ & $\begin{array}{l}\text { Part 2: Italian, Spanish, and Portut } \\
\text { guese Literatures. }\end{array}$ & $\begin{array}{l}\text { Parte 2: Literatura italiana, espa } \\
\text { ñola y portuguesa. }\end{array}$ \\
\hline PT & Part 1: GermanLiterature. & Parte 1. Literatura alemana. \\
\hline
\end{tabular}


126 InvestigacónBiblicteedógica v. 16 N o. 33 julio/ diciembre de 2002

Cuadro 1.(Cont.)

Cla ses y subcla ses del Sis te ma de Cla sifica ción LC

\begin{tabular}{|l|l|l|}
\hline Q & Science & Ciencia \\
\hline R & Medicine & Medicina \\
\hline S & Agriculture & Agricultura \\
\hline T & Technology & Tecnología \\
\hline U & MilitaryScience & Ciencia militar \\
\hline V & Naval Science & Ciencia naval \\
\hline Z & Bibliography and Library Science. & Bibliografía y bibliotecología \\
\hline
\end{tabular}

De manerageneral, losformatosdecadaesquemaobedecen aunaestructurasi milar, detalformaquecadaesquemacuentacon:

1) Prefacio. Proporciona una breve historia particular del esquema, , así como las consideraciones pertinentes a su contenido.

2) Sinopsis. Enlista las subclases contenidas en el esquema

SUBCLASESB-BJ

\begin{tabular}{l|l}
\hline \multicolumn{2}{c}{ SYNOPSIS } \\
\hline $\mathrm{B}$ & PHILOSOPHY (GENERAL) [FILO SO FÍAGENERAL] \\
\hline $\mathrm{BC}$ & LOGIC [LÓGICA] \\
\hline $\mathrm{BD}$ & SPECULATIVE PHILO SO PHY [FILO SO FÍAESPECULATIVA] \\
\hline \multirow{3}{*}{ BF } & PSYCOLOGY [PSICOLOGÍA] \\
\cline { 2 - 2 } & PARAPSYCOLOGY [PARASICOLOGÍA] \\
\cline { 2 - 2 } & OCULT SCIENCES [CIENCIAS OCULTAS] \\
\hline BH & AESTHETICS [ESTÉTICA] \\
\hline BJ & ETHICS [ÉTICA]
\end{tabular}

3) Plan general del esquema. Presenta las subclases y las divisiones más importantes. 
B

69-5739

290-300

290-301

290-302

290-303

290-304

$\mathrm{BC}$

$\mathrm{BD}$
PHILO SOPHY (GENERAL)

History and systems

Including individual philosophers and schools of philosophy

By period

Ancient

Medieval

Renaissance

Modern

LOGIC

By region or country

4) El esquema propiamente dicho. D esarrolla todos los temas y disciplinas que se incluyen en los diferentes números de clasificación.

SUBCLASSES B-BJ

B

PHYLOSOPHY (GENERAL)

Modern (1450/ 1600- )

By region or country

Germany. Austria (German)

By period

Later $18^{\text {th }}$ and early $19^{\text {th }}$ centuries

Individual philosophers

5)Tablasauxiliares. Apoyan la asignación de la clasificación, haciendo más específico su nú mero deacuerdo con laobra, suau tor, su tema, suáreageo gráfica o su forma. 
INDEX TO SUB CLASSESB-BJ

\begin{tabular}{|c|c|c|c|}
\hline \multicolumn{4}{|c|}{$\begin{array}{c}\text { PHYLOSOPHY(GENERAL) } \\
\text { TABLESOFSUBDIVISIONS } \\
\text { (Phi lo sophers with 4,5,9 or } 50 \text { num bers) }\end{array}$} \\
\hline $\begin{array}{c}\text { Table } 1 \\
\text { (50 nos.) } 1\end{array}$ & $\begin{array}{c}\text { Table } 2 \\
\text { (9 nos.) }\end{array}$ & $\begin{array}{c}\text { Table } 3 \\
\text { (4 or } 5 \text { nos.) }\end{array}$ & \\
\hline 0 & 0 & 0 or 5 & $\begin{array}{l}\text { Periodicals. Societies. } \\
\text { Serials }\end{array}$ \\
\hline 1 & 1 & $00 . A 1 A-Z$ & $\begin{array}{l}\text { Dictionaries } \\
\text { Collectedworks }\end{array}$ \\
\hline 3 & 2.A2 & .A2 & $\begin{array}{l}\text { Original(vernacular) } \\
\text { texts. By date }\end{array}$ \\
\hline 4 & .A3 & .A3 & $\begin{array}{l}\text { Editions with commentary. } \\
\text { Byeditor }\end{array}$ \\
\hline
\end{tabular}

6)Índice. Muestrael pano ramageneral deunaclaseypro porcio naacceso alainformación para ubicar la obra en un contexto dado.

SUBCLASSES B-BJ

A

A priori (Epistemology): BD181.3 Achievement motivation

Abbagnano, Nicola: B3611.A23

(Psychology)

Abbt, Thomas: B2632

Abelard (Pierre Abailard):

Child psychology: BF723.M56

B133.A35+

Ackley, H.A. (Spirit messages):

BF1311.A2

7) Páginas suplementarias de adiciones y cambios. Estas páginas incluyen las actualizaciones al Sistema o las nuevas áreas que han surgido. ${ }^{9}$

Como ya se mencionó, los esquemas están conformadospor clases, subclases, divisionesysubdivisiones; las clasesylassub clasesestán estructuradasconletras mayúsculas, mien trasquelasdivisionesysubdivisionespornúmerosarábigosenterosydecimales.Porejemplo:

9 cfr.LiceadeArenas,Judith. "Sistemadeclasificación "LibraryofCongress", tex to programado". En CuadamosdeFilosfía y Ledras Biblideedoǵa no. 9. p. 72. 
B

CLASE
$\mathbf{F}$

SUBCLASE
504

DIVISIÓN
.3

SUBDIVISIÓN

Cadaesquemasi gueunaestructu rajerárquicaen sucon tenido, porlo queunadivisiónestásupeditadaalasubclaseyéstaasuvezalaclase.

Losasuntosespecíficoso temasquesonabordadosporalgunaobrasedividen en subdivisionesotemasdeformanuméricasiguiendounordenprogresivo.

D espuéslaex pan sión detemasaún másespecíficossehacepormedio dedecima les, como en el caso siguien te:

$\mathrm{HA}$

STATISTICS

Theory and method of social science statistics

31 Tabular and graphic methods

15 Forms

$.2 \quad$ Sampling

.3 Regression and correlation

$.5 \quad$ Hypothesis testing

.7 Estimation

.9 Decision theory

Lasdivisio nesinicial mentesehacían porclases, sub clasesytemassiguien do lacla sificacióndesietepuntos,ideadaporMartellysonlassiguientes:

1) Subdivisionesdeforma. Paraestasub divisiónsecon sideraelformato físico en

el que se presentalain formaciónin cluyen do también los con gresosytrabajosde sociedades. Por ejemplo:

\author{
LAW (GENERAL) \\ FORM DIVISIONS \\ TABLE 1 \\ (5 numbers)
}

1. Bibliography

.2 Periodicals

Class here periodicals consisting primarily of informative material

(newsletters, bulletins, etc.) relating to a particular subject

For periodicals consisting predominantly of legal

Articles, regardless of subject matter and Jurisdiction, see K 1

$.4 \quad$ Monographic series

Intergovernmental congress and conferences

Including legal publications of governing and

Sponsoring organizations, bodies, bureaus, etc.

For other congress, meetings, etc. See 4.6 
2) Teonía. Filosofía.Enestadivisión seempiezaaconsiderarelcon tenido intelectual delosdo cumen tosy aquíse co lo can losmaterialesquecon tienen lateo ríade las diferentes disciplinas. 10

3) Historia. La historia es la disciplina que se puede dividir con más facilidad, de manera cronológica y/ o geográfica. Por ejemplo para la división cronológica:

(155)

159

161

163

167

169
History

Primitive, see GN438+

Ancient

General works

Egipt

Phoenicia

Assyro-Babylonian Empire. Persia

Other O riental

Por otra parte,ladivisióngeo gráficatambién puededarsedemaneraalfabéticao deacuerdo con un orden depreferen cia. Cabeseñalarnuevamen tequelaestructura seharealizado conformealasnecesidadesdeorganizaciónbiblio gráficadelaBibliotecadelCongresoyporello existenestasvariaciones.

Seusael ordenalfabético, cuan do elnúmero delaclasequein dicaladivisión geográficaseñaladicho orden, porejemplo:

By region or country, A-Z.

4) Tratados. Obras generales. Es una división usada para las obras de comprensión general, aunque su cobertura sea de una disciplina en particular, subclase 0 K tema, por ejemplo.

LAW K

Comparative law, International uniform law

Private law - Continued

$618 \quad$ Cases

Interpretation of international uniform law

$619 \quad$ General works. Treatises

5) Derecho.N ormas. RelacionesdeE stado. Este apartado o disciplina está cubierto actualmente por la Cla se K, en la que se in tegran to das las ra mas del dere cho excepto el D erecho internacional que se encuentra en la clase JX.

10 cfr. MartínezArellano, Felipe.qpat p. 16. 
6) Estudioyenseñanza. En esta división se integran las obras sobrelaenseñanza de las diferentes disciplinas que aparecen en el esquema, por ejemplo.

HD ECONOMIC HISTORY AND CONDITIONS

PRODUCTION

Collected works (nonserial)

30 Several authors

Study and teaching. Research

Cf. And HF1101+, Commercial education

.4 General works

.412 Audiovisual aids

.413 Problems, ejercises, examinations

.42 By region or country, A-Z

Museums. Exhibitions, see T391+

7)Materiasysubdivisionesdemateria. En estadivisión sedescribeeldesarro llo in dividual o ex pan sio nesdelas clases, sub clasesytemas. Losespecialistas delas diferen tesdisciplinasseen cargaronderealizarun orden ló gico quedebeserusa do y el cual consiste en ir -dentro de cada disciplina- de lo generalalo particular, por ejemplo:

LIBRARY OF CONGRESS CLASIFICATION SCHEDULES COMBINED WITH ADDITIONS AND CHANGESTROUGH 1988

$\mathrm{RB}$ PHATOLOGY

135 Manifestations of disease - Continued Atrophy

Cf. RL391, Dermatology

Mineral infiltrates, concrementes, etc.

137 General works

138 Calification

Disordes of growth. Hipertrophy, hiperplasia, etc.

Cf. RC648+, Endocrynology

RJ135, Pediatrics

RJ482.G 76, Pediatrics

RL401, Dermatology

140 General works

.3 D warfism

Cf. G N69.3+, Antropology

.5 Gigantism 


\section{Subdivisión temática por país}

D en tro del SistemadeClasificaciónLC, se da la división deladisciplinayden tro deellaseproporcio naladivisióngeo gráficaporregióno país.Porejemplo:

\section{CLASS S}

SB PLAN CULTURE

History and conditions

71

Cf. SB 451 - 479, Gardens and gardening

73

Antiquity

75

77

79

83

85

87 G eneral Cf. GN799.A4, O rigin and prehistory of agriculture Greeks Romans Middle Ages By region or country United States

General works

By region or state, A-Z

Under each state:

.x General works

.x2 Local, A-Z

$O$ ther regions or countries, $A-Z$

Under each country :

.x General Works

.x Local, A- Z

PorotraparteenelSistemadeClasificación LC existen unaseriedeno tas queapoyansuuso.Estasno taspuedenseragrupadasdelasiguienteforma:

\section{NOTAS}

a) No tas de alcance. Son aquéllas quepro porcio nan in formación acercadelacobertura y límites que tiene un número determinado. 
LIBRARY OFCONGRE SSCLASSIFICATIONSCHE DULES

COMBINED WITHAD D ITIONSAND CHAN GESTROUG H 1988

$\mathrm{RB}$ PATHOLOGY

144 Disordes of circulation and balance Including drospsy, edema, embolisma, hemorrhage

Hyperemia, infarction, ischemia, thrombosis

CF. RC630, Metabolic diseases

RC666+, diseases of the circulatory system

b) Notas explicativas. Se usan para identificar algunos conceptos relacionados con el temaquesetrata; detallan lostérmi nosquepuedenserin cluidos en laclase.

\author{
LIBRARY OFCONGRESSCLASSIFICATIONSCHE DULES \\ COMBINED WITHAD D ITIO NSAND CHAN GESTRO UG H 1988
}

Authors

Literary subjects. Scenes from the works of great

Illustrations by several artists, accompany

The text, are to be clasified preferably is

PA-PT; when text is secondary in importance

Illustrations are to be classified in N-Nk

Illustrations after the drawings or paintings

Of special artists are to be clasified

Under the artist in NC, ND, or NE, as the case may be, or in PA- PT

under the author illustrated. In the case of authors such as

Shakespeare, D ante, and G oethe clasiffication in literature is to be preferred

O ther special subjects (alpha betically)

Classes here general works on the topics as well as works on the topics in a particular region, country, etc.

c) Notasderelación. En estasno tas semuestran las rela cio nescon otras clasifica cio nesgenerales o específicasquepueden servirdeguíaparaubicardeformamás adecuada el material. 
LIBRARY OFCONGRESSCLASSIFICATIONSCHEDULES COMBINED WITHADDITIONSAND CHAN GESTRO UG H 1988

$\mathrm{M}$

MUSIC

Sacred vocal music - Continued

Liturgy and ritual

Class here service music officially. Prescribed and fixed by a Church for its Liturgy and ritual in office books, e.g. the G radual chants of the Roman Catholic Church. For other church music (music composed to sacred texts for use in church services) class as provided in the schedule, e.g. Protestant Episcopal Choral Service by one or more composers, M2016; a Te D eum with orchestral accompaniment, M2020; a Credo with organ accompaniment, and latin text, M2079.L3; with English text, M2079.E3; a Magnificat for men's and boys'voices unaccompanied (English text), M2079.E6; hymnals, etc., M2115+; historical Publications, M2. The arrangement is chronological unless otherwise estated For manuscripts and publications mainly rubrical with the music merely one of the liturgical functions, seeBL+

2147 Roman Catholic Church

d) No tas de véa se ( cancelado por la Biblioteca del Congreso a una clasificación vigente.

CLASS S

SB PLANT CULTURE

CF. HG 9968, Crop insurance

Q K, Botany

SD , Forestry

For soil aspects of particular crops, see S597;

Machinery for particular crops, see S715;

Fertilizers for particular crops. see S665;

diseases of particular crops, see SB608;

climatc aspects of particular crops, see

the crop, e.g. SB191.M2, Corn

e) N otas deinclusión. In dican lostemas, regio nesgeo gráficaso paísesquede benserincluidosen eltemaqueseestátratando. 
LIBRARY OFCONGRE SSCLASSIFICATIONSCHE DULES COMBINED WITHAD DITIONSAND CHAN GESTROUG H 1988

$\mathrm{CD}$ DIPLO MATICS. ARCHIVES. SEALS

$\begin{array}{ll}101-102 & \text { Europe } \\ 105-106 & \text { Great Britain } \\ 109-112 & \text { England } \\ 113-116 & \text { Scotland } \\ 116.1-4 & \text { Wales } \\ 117-120 & \text { Ireland } \\ 121-124 & \text { Austria } \\ 124.5-8 & \text { Czechoslovakia } \\ 125-128 & \text { Hungary } \\ 131-134 & \text { France } \\ 135-138 & \text { Germany } \\ & \text { Including West G ermany }\end{array}$

f) N otas de referencia a una división (dividedlike). Son usadas cuando dentro de un número de clasificación existe un rango y en él se indica que se divida como uno anterior. En mu chos casosno sehace estain di cación, porlo queel biblio te cario ten dráqueasu mirqueseestruc tu racomo el ran go si milarqueseen cuen tra inmediatamente anterior al que se está tratando.

HC

290-299.1

286

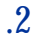

.3

.4

.5

.6

.7

287

288

289

290.5

290-300
ECONOMIC HISTORY AND CONDITIONS Germany

Including West G ermany

20th century

Generals works

World War I, 1914-1918

Reconstrucción, 1919-1945

world War II, 1939-1945

1945-1965

1965-1974

1974-

States, A-Z

Regions, A-Z

Cities, A-Z

Specials topics(not otherwise provided for),

A-Z 2 / .7-.795 East Germany

D ivided like HC270.2-.295

Greece

By period 


$\begin{array}{ll}294 & \text { Byzantine Empire } \\ 295 & \text { Modern Greece } \\ 300.2-.295 & \text { Hungary }\end{array}$

Divided like HC270.2-.295

\section{NÚME ROSDE CUT TER}

Los númerosdeCuttersonusadosden tro del SistemadeClasificación LC para mantenerunorden alfabético quepermitalaiden tificación deun autoren particular, un tema, una región geo gráfica, nom bresdetribuso defamilias devegetaleso deanimales.

LaBiblio tecadelCongreso deEstadosUnidosutilizaunaTabladeCutteradapta daporsuperso nal (véaseanexo 1), queseusaigual quelaTablaoriginal (laideadapor CharlesAmmiCutter)asignán do levalo resnuméricosalasletrassiguientesalainicial delasien to prin cipaldelautor, eltemao laregióngeo gráfica,según seaelcaso.

Por ejemplo, para las obras de 0 ctavio Paz, se asigna el Cutter.P3, aun quecabe aclararquelaaplicación deestatabladepen dedelaco lec ción específicaqueseesté organizan do ydelosau to resqueestén an tesydespuésdeO c tavio Paz, porlo queel número puedevariaralseguirelprincipio delarregloalfabético.

Existen diferen testiposdenúmerosdeCutter: el sim ple, queseaplicaal autorde unaobraen particular; eldo ble, aplicableaalgu nassub divisio nesgeo gráficas, literariasytemáticas, yelCuttersucesivo, queseaplicatam biénaalgunasregio nesgeo gráficas, dan do elnúmero queco rrespon deyanexan do númerosdeformadecimal para suarregloalfabético, lo queseexplicaráenelsiguientecapítulo.

\section{TABLAS AU XILIARESDEL SIST EMA DE CLASIFICACIÓN LC}

Paraenten deradecuadamenteeluso delSistemadeClasificación LC esnecesario co no cerlostiposdetablasysuuso, paraaplicarlasamaterialesqueno necesariamente se encuentran en las fuentes auxiliares que nos proporciona la misma Biblioteca del Congreso. Por ello una amplia explicación ayu daalacom pren sión, aplicacióny adaptación(sifueseelcaso)delsistema.

Lanotaciónusadaenlastablas, deformageneralsedadedosmaneras. ${ }^{11}$

a) Con número de Cutter y;

b) Con números arábigos.

D eacuer do con el uso queseles puededar, las tablas seclasi fi can delasi guien te manera:

11 cfr. Immroth, John Philip. Op at. p.75. 


\section{Tablasdeaplicacióngeneral}

* División geográfica en un arreglo alfabético

* Tablas biográficas

* Tabla de traducciones

Tablasdeaplicaciónlimitada.

* Tablas de autor

* Tablas de forma

* Tablas geográficas

* Tablas para subarreglo interno

Las tablas de aplicación general son algunas tablas geográficas que tienen como baseun número deCutter, al igual quelastablas bio gráficasylastablas detraducción; mien trasquelastablasdeapli cación li mitadason aquéllas queseen cuen tran en cada uno delosesquemasysonaplicablesso lamen teaunaclaseo subclase, como pueden serlastablasdeautordelaclaseP, lasdeformadelaclaseK ylasgeo gráficas delac laseHyS.Finalmenteseen cuen tranlastablasparasubarreglo in terno, queseen cuentranenalgunosesquemasespecialesparadeterminadostemas.

\section{TABLAS DE APLICACIÓN GENERAL}

\section{División geográfica en un arreglo alfabético}

Estastablasseusan cuan do seen cuen traen losesquemaslain dicación "Bycountry, A-Z" o "By region or country, A-Z", lo que indica un orden alfabético por el nom bre delare gión o del país (se gún se trate) ylaasigna ción del nú mero deCutter co rrespondientealassiguientesletrasdelnombredelpaís.Porejemplo, paralaobra:

Título

Plant buyers guide of seed and lant materials in the trade sucessor to manning's "plant buyers index" / ed. by Edwin F. Steffek

Edición 5\# completely rev.

Imprim. Boston, Massachusetts : The Massachusetts Horticultural Society, c1949

D escr. $\quad 259 \mathrm{p}$.

Tema Jardinería - Directorios - Estados Unidos

Entrada Ad Steffek, Edwin F. , ed.

Entrada Ad Massachusetts Horticultural Society

LecorrespondelaclasificaciónSB450.943 E 7enlaque:

SB 450.943

E7
Corresponde a los trabajos sobre jardinería

Corresponde al número de Cutter del país, asignado a Estados Unidos 
Paraeluso deladivisiónalfabéticaesnecesario definirdosaspectos:

a) Elcriterio deasignación del nú mero deCutter.Es decir, sisecon si deraquelaexpan sión denúmerosesdecimal pararespetarelordenalfabético,ysetratadeuna biblioteca que contiene y prevee obtener una gran cantidad de acervo, es conveniente asignarle dos números al Cutter para abrir el margen de incorporación de material. Por ejemplo para:

\section{Manzanillo, Col. M35 \\ Maravatío, Mich. M37}

Sillegaseunmaterial relacio nado conlaciu dad deMapastepec, sepo dríaasignar el número M36 sin mayor problema.

\section{Manzanillo, Col. M35 \\ Mapastepec, Chis. M36 \\ Maravatío, Mich. M37}

b) El idio maen quesevaaordenarel material.G eneral men teen laaplicación delas tablasquedan aperturaparalaasignación deCutter, yaseageo gráfico o temático, en México yen los países dehablahispanasehaceladesignación en español; sin em bargo siel desglo sedelastablasseen cuen trapreestablecido porel Sistemade Clasificación, que está en in glés, se harespetado esaasignación yseco lo cael nút mero deCutter en in glés, detal forma quese usa demaneramix tay pro vo cadis persión en la organización de la colección.

Porejemplo:

CJ

$$
\text { NUMISMATICS }
$$

Coins

CJ

161 Symbols, devices, etc., A-Z

.A6 A nimals

.E5 Elephant

.F3 Facing

Sialaunidad deinformaciónllegaseunaobraquecon si derelaescaleracomo un símbolo, ¿quénúmero deCutterseleasignaría?E 6en españolo seten dríaquetradur cireltérmino.

Al respecto ymien trasno seestablez caun linea mien to general paralospaísesde habla hispana, convendría señalar una política institucional por escrito de acuerdo conlo establecido porladependenciayten dríanqueconsiderarsedosaspectos:

1) Silaco lec ciónyaestáorganizadayregistradacon esadualidad, seten dráqueestablecerunapo líticacon si deran do lafechaapartirdelacual sevaaaplicarlaforma de asignación del Cutter. 
2) Si es una co lección pororganizar, seconsiderarálaaplicación denúmero deCut terparatemas, regio nesgeo gráficasynom bres detribus, razaso familiasdevege tales, en español.12 Lo anterior implicaría la traducción de los temas, regiones geo gráficas, etcéteraqueestánpre- establecidosenelSistemadeClasificaciónLC Las tablas de división geográfica, organizadas por orden alfabético aparecen en losesquemasC,D ,E-F,HT,UyV.

\section{Regiones}

Paralasregio nesquetienenlain dicación "Byregion orcountry, A-Z", sevaaasig narelnúmero deCutterdeacuerdo conlaco berturadeltema. Porejemplo.

\section{África del Norte .A353}

Paraunaobraquecubrael paísan tesquelaregiónyqueso lamen tehacereferencia a ella. Sin em bargo, siel do cumen to serefierealaregión en particularseleasignael Cutterdeacuerdo conelordennaturalquedebetenerdicharegión, porejem plo:

\section{Asia .A78}

Asia Central .C45

Asia del Sur .S87

Porotraparte, enalgunoscasosparalassubdivisionesdelu gareslo cales, el esque macon tem plaquesehagabajo el país paratrabajos generales, yparaloslugareslo ca lesseleañadaelnúmero 2al primerCutteryseleasignaotro Cutterparalalo calidad:

Porregióno país, A-Z

Bajo cadauno (Losnúmeroslo calesusadossolamentebajopaís)

.X Trabajos generales [ $\mathrm{x}=$ número de Cutter para país]

X2 Local, A-Z

\section{T ablas biográficas}

Lastablas bio gráficasson usadas paralas obrasquetratan so brelavidadeal gu nos perso najesimportan tesen diferentesdisciplinas, o bien paraelrelato delavidadel personaje por sí mismo (auto bio grafía); también sein cluyendo cumen tosescritosporun autorcomo cartas, diariosy en trevistas. Latablaseen cuen traenlaclaseCytradu cida, quedadelaformasiguiente.

.X Cutter para el biografiado

.xA2 O bras en colección. Por fecha

.xA25 Selección de obras. Selecciones. Por fecha Incluyendo citas

.xA3 Autobiografía, diarios, etc. Por fecha

.xA4 Cartas. Por fecha

12 Puedeapo yar, en estesen ti do, el Cuadro deregio nesypaí ses en al fabeto único, queseen cuen tra en el Manual paradmangjoyaplicacoóndelaClasificacióndalCongresoalahistonia, ClasesC,DyE-F, de Roberto Abell B. 
.xA5 Discursos, ensayos y lecturas. Por fecha Incluyendo entrevistas

.xA6-Z* Biografía individual, entrevistas y crítica.

Por asiento principal

Incluyendo crítica de obras selectas, autobiografía,citas, cartas, discursos, entrevistas, etc. 13

Immroth ${ }^{14}$ su gierequeparaasignarleelCutteralas en tradas parabio grafíaycríti caqueinicien conlaletraA, seconsiderenlossiguientesrangos:

$$
\begin{aligned}
& \text { Aa-Af }---------- \text { A6-699 } \\
& \text { Ag-Al--- - - - A A-799 } \\
& \text { Am-Ar - - - - - - - A8-899 } \\
& \text { As-Az- - - - - - A 9-999 }
\end{aligned}
$$

Porejemplo,laobrasiguiente:
Autor
Ruíz Meza, Víctor
Titulo
Altamirano : bocetos juveniles
Imprim. México : D epartamento de Bibliotecas, 1958

D escr. $\quad 93 \mathrm{p}$.

SeclasificaríaenPQ 7297.A6Z86endonde:

PQ 7297 Es la clasificación correspondiente a escritores mexicanos que escribieron en un periodo cuyo límite es 1960.

.A6 Es número de Cutter asignado a la persona biografiada

Z86 Es el número de Cutter asignado al autor de la biografía (Ruíz Meza, Víctor) de acuerdo con la tabla siguiente.

TABLA DE VALORES PARA LITERATOS, BIOGRÁFIA Y CRÍTICA

1a Letra apellido del autor y valor asignado

\begin{tabular}{|l|l|l|}
\hline A Z52 & I Z68 & Q Z84 \\
B Z54 & J Z7 & R Z86 \\
C Z56 & K Z72 & S Z88 \\
D Z58 & L Z74 & T Z9 \\
E Z6 & U Z92 \\
F Z62 & M Z76 & V Z94 \\
G Z64 & N Z78 & W Z96 \\
H Z66 & O Z8 & X Z98 \\
& P Z82 & Y,Z Z99 \\
\hline
\end{tabular}

13 Tablatraducida del esquema de clasificacióncorrespondiente a la clase C.

14 Immroth, John Philip. qpat p. 354. 


\section{T abla de traducciones}

Seusaco mún menteparaarreglarlastraduccio nesdelas obrasysepuedeaplicara cualquierdisciplinao clase. 15

.x Trabajos originales

.x1 2 Políglotas

.x13 Traducciones al inglés

.x14 Traducciones al francés

.x15 Traducciones al alemán

.x16 Traducciones al italiano

.x17 Traducciones al ruso

.x18 Traducciones al español.

Porejem plo, paralaobra:

Autor Marx, Karl, 1818-1883

Titulo

Imprim

El capital / Carlos Marx ; ed. Abreviada por Julian

Borchardt ; con estudio crítico preliminar sobre Marx por

Vicente Vázquez ; tr. D e María Cristina Samatier

Descr.

México : Sánchez Mato, c1981

Tema

$379 \mathrm{p}$

Tema

Capital

EntradaAd

Economía

LecorrespondelaclasificaciónHB501M28718,endonde:

HB 501 Es el número asignado a la obra.

M287 Es el número de Cutter asignado a Marx, Karl y

18 Es el número que se le agrega al Cutter para indicar que es una traducción de El Capital al español.

\section{TABLAS DE APLICACIÓN LIMITADA}

Muchastablasaparecenenlosesquemasquesonaplicablesaunaclaseo subclaseen particular,como son lastablasdeau tordelaClaseP, lasdeformadelaclaseK ylas geográficasenlaclaseH.Aquísein cluyenlastablasdeautor,lasdeformaylasgeo gráficas.

\section{T ablas de autor}

Se usan para organizarlos trabajosdeal gún au toren particu lar, selo calizanen la claseB paralostrabajosdefiló so fosyenlaPparalostrabajosdeescritores.

15 Tiene algunas limitaciones que se señalaran en la tabla correspondientea la clase P. 
Paralosfiló so fos, seestablecen lossiguientesran gosdenúmeros50,9,5,4,1 enlas tablas de subdivisión de la clase B, para separar revistas, diccionarios, textos, obras originales,etcétera.Porejemplo,enelesquemaaparece:

$\mathrm{B}$

PHILOSOPHY (GENERAL)

B

Modern (1450/ 1600- )

By region or country

Germany. Austria (G erman)

By period

Later $18^{\text {th }}$ and early $19^{\text {th }}$ centuries

$3100-3149$

Individual philosophers

Schopenhauer, Arthur (1)

Parailus trar el ran go de 50 nú meros, veamos las obras deArthur Scho penhauer. Parael au torsees tableceen el esquemadelaclase B el ran go B3100- 3149, yparacla sificarlaobrasiguiente:

Autor Schopenhauer, Arthur, 1788-1860

Título Samtliche werke / Arthur Schopenhauer

Imprim. Leipzig : F. A. Broadhaus, 1938-

Descr. $\quad 2$ t. En 1 vol.

Leco rrespon delaclasificación B31031938, usan do latabla1 delaclaseB parafilósofoscon50números(deacuerdo conelrango general).

LIBRARY OF CONGRESS CLASSIFICATION SCHEDULES COMBINED WITH ADDITIONS AND CHANGES TROUGH 1988

B

PHILOSOPHY (GENERAL)

TABLES OF SUBDIVISION

(Philosophers with 4, 5, 9 or 50 numbers)

\begin{tabular}{|l|l|l|l|}
\hline Ta ble 1 (50 nos. $)^{1}$ & $\begin{array}{l}\text { Table2 } \\
(9 \text { nos. })\end{array}$ & $\begin{array}{l}\text { Table 3 }(4 \\
\text { or 5 nos. })^{2}\end{array}$ \\
\hline 0 & 0 & $\begin{array}{l}0 \text { or } 5 \\
\text { A1A-Z }\end{array}$ & $\begin{array}{l}\text { Periodicals. Societies. } \\
\text { Serials } \\
\text { Dictionaries } \\
\text { Collected works } \\
\text { Original(vernacular) } \\
\text { Texts. By date } \\
\text { Editions with comentary. } \\
\text { By editor }\end{array}$ \\
\hline & 1 & A16A-Z \\
\hline
\end{tabular}




\begin{tabular}{|l|l|l|l|}
\hline 5 &. .45 & .A5 & Partial editions, selections, etc. By date \\
$15-44$ & 3 & 1 or 6 & Separateworksalphabetically.Bytitle \\
48 & 7 & & Critics and interpretation \\
\hline
\end{tabular}

D e tal forma que, sustituyendo valores, queda la clasificación asignada ya que es untex to enlen guaoriginalalqueseleañadelafechadepublicación(bydate).

A una selección de textos de la obra de Schopenhauer se le asignaría el número B3105.

Autor Schopenhauer, Arthur, 1788-1860

Titulo Briefe an Becker, Frauenstadt, v. D oas, Linnder und Asher.....

Imprim. Leipzig : P. Reclam jun, 194- .

D escr. $223 \mathrm{p}$.

Endonde:

B 3100 Es el número de clasificación de inicio del rango.

+ 5 Es el número indicado en la tabla de 50 números, para la selección de obras (Selections).

B3105 1940. ${ }^{16}$ y se ordenen por fecha (bydate).

Paralacríticaein terpretación delasobrasdeScho penhauer, seleasignaríalaclasificaciónB3148.

Autor Guita, Pedro

Titulo Schopenhauer e kardec : contrastes e confrontos

Imprim. Sao Paulo : Gráfica Urupes, 1970

D escr. $230 \mathrm{p}$.

Endonde:

B 3100 Es el número de clasificación de inicio del rango

+ 48 Es el número indicado en la tabla de 50 números, para la crítica e interpretación.

Parafiló so fosquetienen asignadosran gos de4ó 5 nú meros, seusalatabla3 desubdivisiones, delasiguienteforma:

16 Aquíesnecesario aclararquelasseleccio nessonarregladasporfechadepublicacióny como al autorseleasignaun nú mero declasi ficación, yano seusael nú mero deCutterdeau tor,el arre glo es porfecha. 
Para los trabajos realizados por Vico, Giambattista, se le asigna un rango de B3580-3583, detalformaqueparalaobra:

Autor Vico, Giambattista 1668-1744

Titulo Principios de una ciencia nueva en torno a la naturaleza comun de las naciones / G iambattista vico ; prol. y tr. de José Carner

Imprim. México : El Colegio de México, 1941

D escr. $\quad 2 \mathrm{v}$

EntradaAd Carner, José, prol.

EntradaAd El Colegio de México

Se le asigna la clasificación B3581P73 S62, en la que:

B3580 Es el inicio del rango establecido en el esquema.

$+1 \quad$ Lo establece la tabla 3 para las obras separadas.

P73 Número de Cutter, asignado al título traducido.

S62 Número de Cutter para el idioma en que se realizó la traducción (Spanish).

Paralosescrito resseusan lastablas deformasimilaralas delosfiló so fos, sin embargo tienen ciertas particularidadesque se detallan a continuación, ejemplificando conobrasdeescritoresmexicanos. ${ }^{17}$

Las tablas des tinadasadesarro llarlosblo ques decadauno delospaíses en laliteraturalatino americanason tres. Laprimeradeellas, lamásgrande, es destinada para aquellos países a los que se les asignan bloques de 200 números y es [la Tabla XX]. La siguiente, la tabla [XXIII], es para aquellos otros con bloques de 20 números y la última, la más pequeña [XXIV] está dedicadaaaquellospaísesaloscualesúnicamen teseles han dado bloques de 10 números. 18

Depen dien do delrango quetengaasignado elpaísydel tipo dematerial queseesté clasifican do, sevaade termi narlatablaquesevaausar. Porejem plo paralaobrasiguiente:

Título

Presente de navidad : cuentos mexicanos del siglo XIX / prol. de Jorge Ruedas de la Serna ; selección de Jorge Ruedas de la Serna y Celia Miranda Carabes

Imprim. México : UNAM, Coordinación de Humanidades, Dirección General de Publicaciones, 1994

D escr. $\quad 146$ p. : il.

Serie Ida y regreso al siglo XIX

ISBN 968-36-3572-5

Tema Cuentos mexicanos - Colecciones

17 En 1988 se publi có laClassP. Sub clas sesP-PZ.LanguageandLiteratureTables.En las ta blas se hacen al gunasmo dificacio nesrespec to al nú mero y con tenido deunascuan tasqueseconsideran en el anexo 2 de este trabajo.

18 MartínezArellano,Felipe.Op at. p. 93. 
Tema Cuentos de navidad - Colecciones

EntradaAd Ruedas de la Serna, Jorge, prol.

EntradaAd Miranda Carabes, Celia, comp.

EntradaAd Universidad Nacional Autónoma de México. Coordinación de Humanidades.

El rango que establece el esquema para la literatura de México es de PQ 7100 a PQ 7295, porlo que se usa la tablaXX, su mán do leal nú mero ini cial del ran go, el que establecelaTabla.

La clasificación que le corresponde es PQ 7285 P74, ya que:

PQ 7100 Es el número inicial del rango para literatura mexicana.

+ 185 Se suma el número indicado en la tabla XX para la selección de cuentos

P74 Es el número de Cutter que se le asigna al título.

Paraunaobradeliteratu racubana, seusalatablaXX III,modificada.Asítenemos queparalaobra:

Autor Llorens, Irma

Titulo Nacionalismo y literatura

Imprim. Lleida : Universital de Lleida, 1998

Descr. $283 \mathrm{p}$.

Tema Literatura cubana - Historia y crítica Usando la tabla XXIII y sustituyendo valores:

PQ7370 Número inicial del rango para literatura cubana

+2 Se suma el número indicado en la tabla

D acomo to tal:PQ 7372y yeasignanú mero deCutterparael autordelaobra:L56.

Paralas obras deliteraturadeGuatemala, seusalatablaXXIV mo dificada.D etal formaqueparalaobra:

Autor Johnson, Esther Musson

Titulo The motif of the nahual in guatemalan prose fiction

Imprim. Ann Arbor, Michigan : University Microfilm,

D escr. $\quad 168 \mathrm{p}$

Tema Literatura guatemalteca

Considerando que el esquemamarcaun ran go dePQ 7490aPQ 7499.2y susti tuyendolosvalo ressetieneque:

PQ7490 Número inicial del rango para literatura guatemalteca

+ 5 Se suma el número indicado en la tabla XXIV, para drama. Da como total: PQ 7495 y se asigna número de Cutter al autor de la obra: J65.

A losescritoresindividualesselesasignaunCutterespecífico, elesquemamuestra generalmen tealosmás co no cidos, porlo quelos escrito resqueno han sido registra dosen elEsquemasedeben ubicaren el orden alfabético co rrespon dien teyhayque 
asignarlesunnúmero deCutterúnico quepermitaubicarsupro ducciónliteraria.Generalmen tealosescrito resselesasignaunnúmero declasificación conbaseen el perio do en el cual realizó su mayorpro duc ciónyden tro del perio do el nú mero deCut ter específico. Por ejemplo, para las obras de escritores mexicanos del periodo com prendido en tre1810/ 25, selesasignaelnúmero declasificación PQ 7296yaJua naInés delaCruz, seleasignael Cutter.J6; paraasignarleclasificación asusobras se usalatablaX L, delasiguienteforma.

Paralaobra:

Autor Juana Inés de la Cruz, Sor, 1651-1695

Titulo Los empeños de una casa / Sor Juana Inés de la Cruz ; prol. de Julio Jiménez Rueda

Imprim. México : UNAM, 1940

Descr. $199 \mathrm{p}$

EntradaAd Jiménez Rueda, Julio 1896, prol.

Elnúmero asignado aSorJuanaesPQ 7696.J6, deacuerdo con latablaXL, lostra bajosindividualesseordenanportítulo, delaformasiguiente:

Trabajos individuales. Por título...... xA61-z458

Lo quenosin dicaquelax sesustituyecon el número deCutter.J6,ylostítulosde susobrasseordenan alfabéticamen teapartirdelCutterA61 hastaZ458. Paralaobra queseestáclasificando, setiene:PQ 7296.J6E 5

Respecto alosauto reslatinoamericanoscontem po ráneos, nosencontramosque selesasignaun rango denúmerosdecimales; porejem plo alosescrito resmexicanos cuyas obras se dieron a conocer a partir de 1960 a la fecha, se les asigna el número PQ 7298aPQ 7298.36. Paraasignarel número declasificación adecuado seusalata blaXXIX.

SUBCLASES P-Z LANGUAGE AND LITERATURE TABLES

LITERATURE

TABLES OF SUBDIVISIONS - CONTINUED

TABLE XXIX

The autor number is determined by the second letter of the name

Each author is subarranged by table X L, unless otherwise especified 


$\begin{array}{ll}.13 & \mathrm{C} \\ .14 & \mathrm{D} \\ .15 & \mathrm{E} \\ .16 & \mathrm{~F} \\ .17 & \mathrm{G} \\ .18 & \mathrm{H} \\ .19 & \mathrm{I} \\ .2 & \mathrm{~J} \\ .21 & \mathrm{~K} \\ .22 & \mathrm{~L} \\ .23 & \mathrm{M} \\ .24 & \mathrm{~N} \\ .25 & \mathrm{O} \\ .26 & \mathrm{P} \\ .27 & \mathrm{Q} \\ .28 & \mathrm{R} \\ .29 & \mathrm{~S} \\ .30 & \mathrm{~T} \\ .31 & \mathrm{U} \\ .32 & \mathrm{~V} \\ .33 & \mathrm{~W} \\ .34 & \mathrm{X} \\ .35 & \mathrm{Y} \\ .36 & \mathrm{Z}\end{array}$

D eestaformael número seasignadeacuerdo conlaprimeraletradelapellido del autoryelNúmero deCutterdeacuerdo conlaletrasiguien te; el su barreglo delaobra sehaceconsiderandolosparámetrosdelatablaXL.Asíparalaobra:
Autor
Hernández, Lorenzo
Titulo
Para el mediodía / Lorenzo Hernández
Imprim.
México, D. F. : SEP : Crea, 1988
D escr.
$70 \mathrm{p}$.
ISBN
968-29-1997-5
Lasignaturatopográficaqueda:PQ 7298.18E4P37
PQ7298
El número base de clasificación asignado por el esquema.
.18
E4
La adición de acuerdo con el asiento principal del autor.
El número de Cutter asignado de acuerdo con la letra siguiente del asiento principal.
P37 El número de Cutter para el título específico.

Endonde: 


\section{T ablas de forma}

Lastablasdeformamuestran cómo debenorganizarselosmaterialesbiblio gráfi cosapartirdesu estructurafísicao biblio gráfica; es decir, apartirdequesean publicaciones periódicas, colecciones, enciclopedias, anuarios, directorios o publicaciones oficiales, en treotras. Estastablas aparecen en laclaseK yseusan delasiguien teforma:

Paralaobra:

Autor Lowenfeld, Andreas F., 1930-

Título International litigation and the quest for reasonableness : essays in private international law / Andreas F. Lowenfeld

Imprim. Oxford : Clarendon; New York : Oxford University, 1996

Descr. $\quad 243 \mathrm{p}$.

ISBN 0198260598

Tema Procedimientos civiles (D erecho internacional)

Tema Jurisdiccion (D erecho internacional)

Tema Conflicto en leyes

EntradaAd Oxford University

A lostrabajosgeneralesdepro cedimien to civilin ternacio nal, selesasignaun rango deK 7611aK 7615yseremitealaTabla1.

Conflict of laws

\section{LAW(GENERAL)}

Intelectual Property

$7580 \quad$ Unfair competition

7582 General works

Special topics, A - Z

Civil procedure. International civil procedure

7611 - 7615 General (I)

7624 labor law

Judicial assistance (II)

For letters rogatory, see K 7673; enforcement of

Foreing judments, see K 7683+

Jurisdiction. Acces to courts

Y seleasignaríalaclasificacióndeacuerdo conelsiguientepro cedimiento:

K7610 Clasificación inicial del rango

15.6 Número de la división de forma, que incluye trabajos generales de

procedimiento civil internacional y que sustituye los dos últimos

dígitos del rango.

K7615.6 Número de clasificación asignado a la obra. 
LatablaII dedivisio nes deformadelaclaseK (D erecho general) esparalas obras conunnúmero declasificaciónespecífico.Porejemplo, paralaobra:

Autor Balleste, Cristina

Titulo El divorcio y la adopción / Cristina Balleste

Imprim. Barcelona : D ecálogo, 1990

D escr. $142 \mathrm{p}$

ISBN 84-87404-09- $\mathrm{x}$

Tema Divorcio

Tema Adopción

Leco rrespon delaclasificación K 695ysehaceel en vío alaTablaII.Como setrata deuntrabajo generalso breeldivorcioylaadopción, seremitealapartado:

.A 7-Z8Trabajosgenerales.Tratados

Lo quein dicaquelasobrasgeneralessedeben organizarporasien to prin cipal, de manera alfabética sin usar de la A1-A6. En el caso de la obra presentada, el asiento principal esparaBalleste,Cristina, porlo queno haynin gún pro blemaparalaasigna cióndelCutter.

Cadasub clasedelaclaseK (D erecho) tienesustablas dedivisio nesdeformayse usan igual. ${ }^{19}$

\section{T ablas geográficas}

Existen dostiposdetablasgeo gráficas. Elprimero deacuerdo con un orden al fa bético queyafueex plicado enlastablasdeusogeneral.

Elsegun do tipo esun arreglo clasificado en elquelasáreas geo gráficastienen asig nados números específicos dentro de la clasificación. Ejemplos de estas tablas, las encontramosenlaclaseS(Agricultura), $\mathrm{H}$ (Cienciasso ciales)yN(BellasArtes).

Porejem plo, paralaobra:

Autor Abrego Ayala, Jesús O ctavio

Título Corporaciones publicas: un diseño organizacional para fortalecer su gestión / J. O ctavio Abrego Ayala, Silvano Barba Behrens

Imprim. México, D . F. : Instituto Nacional de Administración Publica, 1989

Descr. $\quad 398 \mathrm{p}$

ISBN 968-6403-00-0

Tema Empresas gubernamentales - México

Tema Empresas comerciales del gobierno - México

EntradaAd Barba Behrens, Silvano, coaut.

Seleasignaunran go deHD 4001-4420.7, enelqueseremitealatablaIX .

19 Véase el Manual para d manjoy aplicaión dela Clasificaión di Congresoal Deeeho Eurqueosubdases $\mathrm{KJ}-\mathrm{KKZ}$, de Ro berto Abell. 
Como sepuedeob servaren latabla, aMéxico seleasignaun ran go de 5 nú meros (11-15).Suman do estosdígitosal rango inicial, laobraqueseestápro cesan do ten dría queestarentreHD 4011-4015. Sevuelvealapáginaen queseasignó el primerrangoy seusalatablaquein dicaelarreglo bajo cadapaís.

\begin{tabular}{|c|c|c|c|}
\hline \multicolumn{4}{|c|}{$\begin{array}{l}\text { ECONOMIC HISTORY AND CONDITIONS HD } \\
\text { stry }\end{array}$} \\
\hline $4001-4420.7$ & $\begin{array}{l}\text { Other regior } \\
\text { Under ea }\end{array}$ & $\begin{array}{l}\text { intries. Tal } \\
\text { ntry }\end{array}$ & \\
\hline$\frac{10 \text { nos. }}{(1)}$ & $\frac{5 \text { nos. }}{(1)}$ & $\frac{1 \text { nos.. }}{\text { A1 -5 }}$ & $\begin{array}{l}\text { Periodicals. Societies. } \\
\text { Serials }\end{array}$ \\
\hline $\begin{array}{l}\text { (5) } \\
(7)\end{array}$ & (3) & $. \mathrm{A} 6-\mathrm{z} 7$ & $\begin{array}{l}\text { G eneral works } \\
\text { O rganization and } \\
\text { administration }\end{array}$ \\
\hline (8) & (4) & & Public policy \\
\hline (10) & (5) & $. Z 8 A-Z$ & Local, A - Z \\
\hline
\end{tabular}

Seeligeelblo quede5númerosysesustituye, detalformaquealaobraseleasigna ríalaclasificación:HD 4013

\section{T ablas para subarreglo interno}

Estassonlastablasqueseen cuen tran másfrecuen tementeen el esquemadeclasi ficación LC, en las que se considera un rango de nú merosparaun temaen especial. Estesubarreglo puedeocupartablas deforma, cro no ló gicaso temáticasyseen cuentranen casito doslosesquemas.

Unejem plo deladiversidad decombinacio nes detablas, lo señalaLoisMaiChan, al in di carquelastablas paraarreglo detrabajos deliteratu ra (en general) bajo países, se inicia con PN4901-5600, bajo el rango de 20 núms., la columna de los primeros cuatro números (1-4)represen tadivisio nesdeforma;lossiguien tescuatro números (5-8) son divisio nescro no ló gi cas; losnúmeros 12y13 son ejem plos deformaydivi sión temática; el nú mero 14 parató pi cosespeciales delaA-Z, yesotro tipo dedivisión temática; los números "16-19, Local”, son divisiones geográficas; y el número "20" esunadivisióntemáticaparadividirlarevistasespecializadasyotraspublicaciones, A-Z.20

20 dr. Immroth, John Philip. qp att p. 100. 
JOURNALISM

Journalism.Theperiodicalpress, etc.

Byregionorcountry-Continued

TA BLE FO RARRAN GE MENTOFWORKSUN DERCOUNTRIES

FortheUnitedStates, see PN4841+

20 nos. 10 nos.

0

1

2

3

$\begin{array}{ll}4 & 2 \\ 5 & 3 \\ 4 & \\ 6 & \\ 7 & \\ 8 & \end{array}$

9

12

13

14

7
Periodicals, see PN4701+

Societies, conferences,

Societies collections

Conferences. Congresses

Collections

History and other general works

Comprehensive

Early

Recent

18th Century

19th Century

20th Century

Biography of editors, journalist, etc.

For Historical characters, see D - F

A1 - $5 \quad$ Collective

.A1 - Z Individual, A - Z

A39

A4

A75

.B55

.B75

.C35

. $\mathrm{C} 4$

. $\mathrm{C} 5$

.C57

.66

. $\mathrm{C} 63$

. $\mathrm{C} 65$

.667

. $\mathrm{C} 74$

.D58

.T37
Special topics, A - Z

Advice columns

Agricultural journalism

Arts

Black press

Broadcast Journalism

Ganards

Catholic press

Children's periodiodicals, see J8

Circulation marketing

Commercial Journalism

Communist press

Community newspapers

Correction articles

Country newspapers. Rural Journalism

Crime journalism

Disaster reporting

Technical Journalism 
16

178

199

$\begin{array}{ll}\text {.T4 } & \text { Television journalism } \\ \text {.T43 } & \text { Television program guides } \\ \text {.U5 } & \text { Underground literature } \\ \text {.U53 } & \text { Underground press } \\ \text {.W3 } & \text { Wages } \\ \text {.W36 } & \text { War } \\ \text {.W45 } & \text { Wholesalers } \\ \text {.W58 } & \text { Women } \\ \text {.W6 } & \text { Women's magazines } \\ & \text { Local }\end{array}$

By region, $\mathrm{A}-\mathrm{Z}$

By state, province, etc., A- Z

By place, A-Z

Under each, using successive

En la subclase AM existen tablas para sub-arreglo interno, que merecen especial atención.

Class here general works only. For museums and

Collections in special fields, see classes B-Z

10-100 D escription and history of museums

By country

For individual museums of a general nature, see AM101

Under each country (Three numbers)

(1) General works

(2) States, provinces, etc., A-Z

(3) Cities, towns, etc., A-Z

Under each country (Two numbers):

(1) .A1A-Z Periodicals. Society

A2A-Z G eneral works

A3-Z Provinces, etc., A-Z

(2) Cities, towns, etc.

Undereachcontry(O nenumber)

A2A-Z Generalworks

A3A-Z Statesprovinces, etc.,A-Z

A4-Z Cities, towns, etc.

Undereachcountry(Cutternumber)

x Generalworks

.x2 States, provinces, etc.,A-Z

.x3 Cities, towns, etc., A-Z 
Estastablastam bién pro veenal clasi ficadordeelemen tosparaarreglarlasobras deun país enáreas geo gráficasespecíficas, porejem plo, aMéxico seleasignaun rango de2números, detalformaqueparalaobra:

Autor México. Instituto Nacional de Antropología e Historia.

Título Museo Nacional de Historia : guía sintética.

Imprim. México : Secretaría de Educación Pública, 1944

Descr. $24 \mathrm{p}$.

Tema Museos - México

Tema Museo Nacional de Historia. México.

Se le asigna la signatura topográfica AM23.A2 M48, en la que:

AM23 Es el número de clasificación para la descripción e historia de museos de México.

.A2 Es el Cutter que se asigna a los trabajo generales, en el rango de dos números.

M48 Es el número de Cutter asignado al asiento principal.

Existen tam bién divisio nestemáticasylashistó ricasporfechas, éstasúltimas, ge neralmente son muy breves.Lasdivisio nesdetipo histó rico-cro no ló gico sepueden observarenlaclaseS(Agricultura), porejemplo paralaobra:

Autor Lockhart, J. A. R.

Título Introduction to crop husbandry : including grassland / by J.

Edición 6

Imprim. Oxford : Pergamon, 1988

Descr. $\quad 319 \mathrm{p}$

ISBN 0-08-034201-9 (pasta dura) 0-08-034200-0 (rústica)

Tema Cultivos

Tema Agricultura

Tema Cultivos - Gran Bretaña

Tema Agricultura-Gran Bretaña

EntradaAd Wiseman, A. J. L., coaut. Se le asigna la clasificación SB98 L63 1988

Yaqueenelesquema, seseñala:

SB PLANT CULTURE

General works

Great Britain

97 To 1800

98 1801-

99 O ther regions our countries, A-Z 
O tro caso desubarreglointerno lo representanlostrabajosfilo só ficosdeMéxico, alosqueselesasignaun ran go de 5 nú meros, deB1015-1019, aplican do latablapara 5números, paraeltrabajo:

Autor López Díaz, Pedro

Título Una filosofía para la libertad (la filosofía de Leopoldo Zea) / Pedro López DíazImprim. México, D.F. : Costa Amic, c1989

Descr. $\quad 311 \mathrm{p}$

ISBN 968-400-075-8

Tema Filosofía mexicana

Tema Filosofía latinoamericana Quedaría la siguiente clasificación, B1019.Z4 L66, en donde:

B1015 Es el inicio del rango establecido en el esquema y sustituyendo los valores de la tabla del esquema:

B1019 Es para los trabajos sobre filósofos individuales, organizándolos por asiento principal de la a-z, por lo que:

Z4 Corresponde al número de Cutter asignado para Leopoldo Zea.

L66 Es el número de Cutter asignado al autor de la obra.

Estos son sólo algunos aspectos del Sistema de Clasificiación que apoyarán su comprensión.

ElSistemadeClasificación LC tam bién cuen tacon otrosdo cu men tosparasuma yorcom pren siónyaplicación, como sonlosín dices, elSubjetCatalogingManual:Shdflistingy losmismosregis tros decatalo ga ción delaBiblio tecadel Con gre so quepue den ser vistos a través del CD Biblio-graphic o de forma más actual a través de Internet.

\section{CONCLUSIONES}

Como se puedeobservar, analizarlaestructuradelSistemadeClasificacióndela Biblio tecadelCongreso yco no cereluso delastablasauxiliaresesunpro ceso querequiere de profesionales del área bibliotecológica que dediquen tiempo a la adquisición deestosco no cimientosparaquepuedan aplicarlosco rrectamentealosmateria les bibliográficos de las distintas unidades de información, ya que de la asignación correctadelaclasificacióndependerálaorganizaciónadecuadadelosmaterialesy su uso óp timo. Sin em bargo, el co no cimiento teó rico no essuficien teparaapren derel uso delsistema, lalecturadematerialesyartículosso breLC no lepro porcio naal profesional la certeza de que va aplicar correctamente la clasificación,esta certeza se la dará la prácticaconstante que ten gaen suám bi to pro fesio nalylaactualización pef manenteatravésdelaeducacióncontinúa. 


\section{BiBLIOG RAFÍA}

Abell, Ro bertL. "Aplicación delaclasifica ción delaBiblio tecadel Congreso en las bibliotecas mexicanas, basada en lapo nen ciadel Prof. Pe dro Zamora". En Jornadas Mexicanas de Biblioteconomía (6 : ) VI Jomadas Mexicamas deBildideecnomá: memria. México : AMBAC, 1975. p. 320-325

- - Manual paradmangoyadicacoóndelaClasificacoónddCongesoalahistona, dasesC,DyE-F.México: UNAM,Cen tro Universitario deInvestigaciones Bibliotecológicas, 1987.

Caster, Li llieC. Thedasifie'sgui detodassLC dass H : subdivisiontedniquesfor thescial siences New Y ork : Neal Schuman, c1986. 143 p.

EvaluadóndelosprogramasdkenseñanzaddSistemadedasificacónddlaBiblioteca ddCongresodelosEstadosUnidos(LC): re unión detrabajo, 5-6 deju lio de 1984. Méxi co :UNAM,Cen tro Universitario deInvestigacionesBibliotecológicas, 1979. 25 p.

GarzaÁvalos, MaríaLuisa.Introduccónaladasificacoóndecienảas(daseQ)end Sistema dela Biblicteca da CongeesodeEstados Unidos México : La autora, 1979. 95 p. Tesis (Licenciada en Biblioteconomía) - ENBA, 1979.

Geiger,S. “ LC classification key:acom parison of classesQ -V with theirZ counterparts". En : Referenequartery. V. 21, issue 3, spring. 1982. p. 280-287.

Greenberg, Alan M. AnauthorindaxtoLibraryfCongessClassification:dass P, subdasses PN, PR, PS, PZ. New Y ork : D ekker, c1981. 80 p.

Grout, CatherineWhite.Anexplanation of thetables usedin theschedulesofthe LibraryofCongessClassification: accompaniedbyhistoricalandexplanatoryin troduction New York : School of Library Service ColumbiaUniversity, 1940. $107 \mathrm{p}$.

Immroth, John Phi lip.Immot's guidetothelibraryofCongressClassificadion 4 ed. Englewood, Colorado : Libraries Unlimited, 1990.

La Mon tag ne, Leo E.American librarydassificationswithspedal referencetothe Library of Congess Hamdem : Shoe String, 1961.

LafuenteLópez,Ramiro.Lossistemashiblidtedóǵgesdedasificadón México : UNAM, Centro Universitario de Investigaciones Bibliotecológicas, 1993. 112 p. (monografías; 15)

Library of Congress. SubjectCataloging División. "Esquema de ClasificacióndelaBiblio tecadelCongreso delosEstadosUnidosdeNorteaméri ca" / tr. y pról. de María Teresa Chávez Campomanes. Washington : Unión Panamericana, 1967. 21 p. (CuadamosBildidtedớgocs; 42). 
Library of CongressClassification scheetules, accumlation of additionsanddhanges Detroit : G ale Research, 1974- .

- -. LC Classification autline 4 th ed. Washington : Library of Congress, 1978.

Licea de Arenas, Judith. "Sistema de clasificación Library of Congress : tex to pro gramado". En CuadarnosdeFilosofíayLetras: Biblictedogóa No. 9. p. 53-98

Lincoln, T. "Ethno-linguistic misrepresentations of the Alaskan native lan guages asmi rro red in theli brary of Con gress of catalo ging and clas sification". EnCataloginganddassificadionquarterty. V.7, Issue 3, 1987. p. 69-89.

MartínezArellano,Filiberto Felipe. Algunosproblemasendmangjoyaplicacón dd Sistema deClasificaión LC en nateriales latimoameicanos México, D .F. : El autor, 1979. Tesis (Lic. en biblioteconomía) - ENBA, 1979.

- -. Ampliacóóndela partedeinstitudioneseducativasdeMéxico(LE7-9)dentrode ladasificacoóndelaBibliotecaddCongresa Méxi co :UNAM, Cen tro Uni versitario de Investigaciones Bibliotecológicas, 1983. 50 p.

- -. "Características fundamentales del sistema de clasificación LC". En Cienaiabiblictearia Año 1, no. 2 (dic. 1977) p. 13-25.

Matthis, Raimund E. y D esmond Taylor. AdqptingtheLibrary of Congess Classificationsystem: amanual ofm thodsant tedhiquesforapdicationorconver sion New York : R.R. Bowker, 1971. 209 p.

Menmia dela aratroreunionesnacionales sdbrela nomalización di usoen Méxic ddSistemadbClasificacoóndelaBibliotecaddCongesodeEstadosUnidos(LC). México: UNAM,Centro Universitario deInvestigacionesBibliotecológicas, $1989.96 \mathrm{p}$.

Olson, Nancy B. ConbinedindexestotheLibraryofCongessClassificationsche dules Washington : U.S. Historial D ocuments Institute, 1974.

Ols rud, L. "LCClassification key : a com pari son of classes A-P with their counterparts". En Referenequartely.V.21, Issue 1, fall 1981. p. $72-73$.

PérezVillegas,Constanza.LadaseFdelaClasificacóndelaBibliotecaddCon geso:suadaptacónyaplicaiónparaMéxico México :Laau to ra, 1969.Tesis (Lic. en bibliotecología) - UNAM, Colegio de Bibliotecología.

Rowell, Lois. "Additions and chan ges". En LibraryJaurnal asturyofseleeted LC Classification schedles. V. 94, no. 19, 1969. p. 3975-3977.

Schim mel pfeng, Richard and C.D o nald Cook. TheusedtheLibraryofCon gess Classification Chicago : ALA, 1968. 245 p.

TheuseofLibrarydCongessClassification, proceedingsofthelnstituteontheuseof theLibraryofCongessClassification july 7-9, 1966 / eds. Schimmelpfing, Richard H. y C. Donald Cooks. Chicago : ALA, 1968. 


\section{ANEXO 1 \\ TABLA DE CUTTER}

(1) Después de una vocal inicial

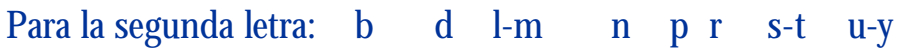

$\begin{array}{llllllllll}\text { Use el número: } & 2 & 3 & 4 & 5 & 6 & 7 & 8 & 9\end{array}$

(2) Después de la letra inicial S

Para la segunda letra: a ch e h-i m-p t $\quad$ u w-z

$\begin{array}{lllllllll}\text { Use el número: } & 2 & 3 & 4 & 5 & 6 & 7 & 8 & 9\end{array}$

(3) Después de la letra inicial $\mathrm{Qu}$

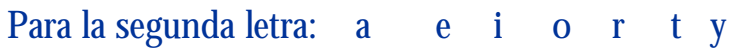

Use el número: $\quad \begin{array}{llllllll}3 & 4 & 5 & 6 & 7 & 8 & 9\end{array}$

Para las letras iniciales Q a-Q t, use:2-29

(4) D espués de cualquier otra consonante

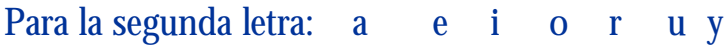

$\begin{array}{llllllll}\text { Use el número: } & 3 & 4 & 5 & 6 & 7 & 8 & 9\end{array}$

(5) Para expansión.

Para la letra:

Use el número:

a-d e-h i-l m-0 p-s t-v w-z

$\begin{array}{lllllll}3 & 4 & 5 & 6 & 7 & 8 & 9\end{array}$

Nota: Esta tabla fue traducida de la que se presenta en la p. 58 de la obra Immoth's guideto theLibrary of Congess Classification 


\section{ANEXO 2 \\ Modificaciones del número de tablas para Lengua, Literatura y Autores individuales}

A continuación se presen tan losnú meros detablas quese usaban ylosnúmeros actuales.Noseconsideraronloscasosenqueno hubo cambio alguno.

\section{TABLAS PARA LENGUA}

$\begin{array}{ll}\text { Número cancelado } & \text { Número actual } \\ \text { Via } & \text { VI } \\ \text { VII } & \text { IX } \\ \text { X } & \text { Cancelada } \\ \text { Xia } & \text { XI } \\ \text { XIII } & \text { IX } \\ \text { Xvb } & \text { XV }\end{array}$

\section{TABLAS PARA LITERATURA}

Número cancelado

$\mathrm{V}$ (PT, Parte 2)

$\mathrm{XX}$

$\mathrm{XXI}$

XXVI

XXVIa

XXVII

XXVIII

XXIXa

XXXa

A

B

C

D

Da

$\mathrm{Da}(\mathrm{PR})$

E

Ea

$\mathrm{F}$

$\mathrm{Fa}$
Número actual

XXIX

Cancelada

Cancelada

Cancelada

Cancelada

XXIV

XXIV

Cancelada

XXX

$\mathrm{XX}$

Cancelada

Cancelada

XXIII

XXIII

XXI

XXIV

XXIV

XXVI

XXV 
TABLAS PARA AUTO RES INDIVIDUALES Y SUS O BRAS

Número cancelado

V (PT, Parte 2)

$\mathrm{XX}$

$\mathrm{XXI}$

XXVI

XXVIa

XXVII

XXVIII

XXIXa

$\mathrm{XXXa}$

$\mathrm{A}$

B

C

$\mathrm{D}$

Da

$\mathrm{Da}(\mathrm{PR})$

E

$\mathrm{Ea}$

$\mathrm{F}$

$\mathrm{Fa}$
Número actual

XXIX

Cancelada

Cancelada

Cancelada

Cancelada

XXIV

XXIV

Cancelada

XXX

$\mathrm{XX}$

Cancelada

Cancelada

XXIII

XXIII

XXI

XXIV

XXIV

XXVI

XXV 